\title{
Integrated Fractional Resolvent Operator Function and Fractional Abstract Cauchy Problem
}

\author{
Ya-Ning Li and Hong-Rui Sun \\ School of Mathematics and Statistics, Lanzhou University, Lanzhou, Gansu 730000, China \\ Correspondence should be addressed to Hong-Rui Sun; hrsun@lzu.edu.cn
}

Received 31 October 2013; Accepted 11 December 2013; Published 8 January 2014

Academic Editor: Yong Zhou

Copyright ( 2014 Y.-N. Li and H.-R. Sun. This is an open access article distributed under the Creative Commons Attribution License, which permits unrestricted use, distribution, and reproduction in any medium, provided the original work is properly cited.

We firstly prove that $\beta$-times integrated $\alpha$-resolvent operator function $((\alpha, \beta)$-ROF) satisfies a functional equation which extends that of $\beta$-times integrated semigroup and $\alpha$-resolvent operator function. Secondly, for the inhomogeneous $\alpha$-Cauchy problem ${ }^{c} D_{t}^{\alpha} u(t)=A u(t)+f(t), t \in(0, T), u(0)=x_{0}, u^{\prime}(0)=x_{1}$, if $A$ is the generator of an $(\alpha, \beta)$-ROF, we give the relation between the function $v(t)=S_{\alpha, \beta}(t) x_{0}+\left(g_{1} * S_{\alpha, \beta}\right)(t) x_{1}+\left(g_{\alpha-1} * S_{\alpha, \beta} * f\right)(t)$ and mild solution and classical solution of it. Finally, for the problem ${ }^{c} D_{t}^{\alpha} v(t)=A v(t)+g_{\beta+1}(t) x, t>0, v^{(k)}(0)=0, k=0,1, \ldots, N-1$, where $A$ is a linear closed operator. We show that $A$ generates an exponentially bounded $(\alpha, \beta)$-ROF on a Banach space $X$ if and only if the problem has a unique exponentially bounded classical solution $v_{x}$ and $A v_{x} \in L_{\text {loc }}^{1}\left(\mathbb{R}^{+}, X\right)$. Our results extend and generalize some related results in the literature.

\section{Introduction}

This paper is concerned with the properties of $\beta$-integrated $\alpha$-resolvent operator function $((\alpha, \beta)$-ROF) and two inhomogeneous fractional Cauchy problems.

Throughout this paper, $\mathbb{R}^{+}=[0, \infty), \mathbb{N}$ denotes the set of natural numbers. $\mathbb{N}_{0}=\mathbb{N} \cup\{0\}$. Let $X, Y$ be Banach spaces, $B(X, Y)$ denote the space of all bounded linear operators from $X$ to $Y, B(X)=B(X, X)$. If $A$ is a closed linear operator, $\rho(A)$ denotes the resolvent set of $A$ and $R(\lambda, A)=(\lambda I-A)^{-1}$ denotes the resolvent operator of $A \cdot L^{1}\left(\mathbb{R}^{+}, X\right)$ denotes the space of $X$-valued Bochner integrable functions: $u: \mathbb{R}^{+} \rightarrow$ $X$ with the norm $\|u\|_{L^{1}\left(\mathbb{R}^{+}, X\right)}=\int_{0}^{\infty}\|u(t)\| d t$, it is a Banach space. $\mathrm{By} *$ we denote the convolution of functions

$$
(f * g)(t)=\int_{0}^{t} f(t-\tau) g(\tau) d \tau, \quad t \geq 0 .
$$

$g_{\alpha}$ denotes the function

$$
g_{\alpha}(t)= \begin{cases}\frac{t^{\alpha-1}}{\Gamma(\alpha)}, & t>0, \\ 0, & t \leq 0,\end{cases}
$$

and $g_{0}(t)=\delta_{0}(t)$, the Dirac delta function.
In 1997, Mijatović et al. [1] introduced the concept of $\beta$ times integrated semigroup $\left(\beta \in \mathbb{R}^{+}\right)$which extends $k$-times integrated semigroup $\left(k \in \mathbb{N}_{0}\right)$ [2], they showed an $R(\lambda)$ to be the pseudoresolvent of a $\beta$-times $(\beta>0)$ integrated semigroup $\{S(t)\}$ if and only if $\{S(t)\}$ satisfies the following functional equation:

$$
\begin{aligned}
\int_{t}^{t+s} & (s+t-r)^{\beta-1} S(r) d r-\int_{0}^{s}(s+t-r)^{\beta-1} S(r) d r \\
\quad= & \Gamma(\beta) S(t) S(s), \quad t, s \geq 0 .
\end{aligned}
$$

In the special case of $\beta=k \in \mathbb{N}$, the corresponding result is summarized in [2].

For the inhomogeneous Cauchy problem

$$
u^{\prime}(t)=A u(t)+f(t), \quad t \in[0, T], u(0)=x,
$$

where $T>0, f \in L^{1}([0, T], X), x \in X$, and $A$ is the generator of a $k$-times integrated semigroup $\{S(t)\}$ on a Banach space $X$ for some $k \in \mathbb{N}_{0}$. Let $v(t)=S(t) x+$ $\int_{0}^{t} S(t-s) f(s) d s, t \in[0, T]$. Lemmas 3.2.9 and 3.2.10 of [2] show that if there is a mild(classical) solution $u$ of (4), then $v \in C^{k}([0, T], X)\left(C^{k+1}([0, T], X)\right)$ and $u=v^{(k)}$. On the other 
hand, if $v \in C^{k}([0, T], X)\left(C^{k+1}([0, T], X)\right)$, then $v^{(k)}$ is also a mild (classical) solution of it.

Furthermore, if $A$ generates an exponential bounded $k$ times integrated semigroup on a Banach space $X$, then, for any $x \in X, v(t)=\int_{0}^{t} S(s) x d s$ is the unique exponential bounded classical solution of the following problem:

$$
u^{\prime}(t)=A u(t)+g_{k+1}(t) x, \quad t \geq 0, u(0)=0 .
$$

In recent years, a considerable interest has been paid to fractional evolution equation due to its applications in different areas such as stochastic, finance, and physics; see [3-8]. One of the most important tools in the theory of fractional evolution equation is the solution operator (fractional resolvent family) [9-15]. The notion of solution operator was developed to study some abstract Volterra integral equations [16] and was first used by Bajlekova [17] to study a class of fractional order abstract Cauchy problem. In [9], Chen and $\mathrm{Li}$ introduced $\alpha$-resolvent operator functions ( $\alpha$-ROF for short) defined by purely algebraic equation. They showed that a family $\left\{S_{\alpha}(t)\right\}_{t \geq 0} \subset B(X)$ is an $\alpha$-ROF if and only if $\left\{S_{\alpha}(t)\right\}_{t \geq 0}$ is a solution of abstract fractional Cauchy problem

$$
\begin{aligned}
& { }^{c} D_{t}^{\alpha} v(t)=A v(t)+g_{\beta+1}(t) x, \quad t>0, \\
& v(0)=x, \quad v^{(k)}(0)=0, \quad k=1, \ldots, N-1 .
\end{aligned}
$$

When $0<\alpha<1$, Peng and Li [18] proved that the solution operator $\left\{S_{\alpha}(t)\right\}_{t \geq 0}$ for (6) satisfies the following equality:

$$
\begin{aligned}
\int_{t}^{t+s} & \frac{S_{\alpha}(\tau)}{(t+s-\tau)^{\alpha}} d \tau-\int_{0}^{s} \frac{S_{\alpha}(\tau)}{(t+s-\tau)^{\alpha}} d \tau \\
\quad & \alpha \int_{0}^{t} \int_{0}^{s} \frac{S_{\alpha}\left(\tau_{1}\right) S_{\alpha}\left(\tau_{2}\right)}{\left(t+s-\tau_{1}-\tau_{2}\right)^{1+\alpha}} d \tau_{1} d \tau_{2}, \quad t, s \geq 0 .
\end{aligned}
$$

We refer to $[5,15,16,19]$ for further information concerning general resolvent operator functions. In addition, Chen and $\mathrm{Li}$ [9] also introduced the concept of integrated fractional resolvent operator function in an algebraic notion as follows.

Definition 1 (see [9, Definition 3.7]). Let $\alpha>0, \beta \geq 0$. A function $S_{\alpha, \beta}: \mathbb{R}^{+} \rightarrow B(X)$ is called a $\beta$-times integrated $\alpha$-resolvent operator function or an $(\alpha, \beta)$-resolvent operator function $((\alpha, \beta)$-ROF for short) if the following conditions hold:

(a) $S_{\alpha, \beta}(\cdot)$ is strongly continuous on $\mathbb{R}^{+}$and $S_{\alpha, \beta}(0)=$ $g_{\beta+1}(0) I$

(b) $S_{\alpha, \beta}(s) S_{\alpha, \beta}(t)=S_{\alpha, \beta}(t) S_{\alpha, \beta}(s)$ for all $s, t \geq 0$;

(c) the functional equation

$$
\begin{aligned}
S_{\alpha, \beta}(s) & J_{t}^{\alpha} S_{\alpha, \beta}(t)-J_{s}^{\alpha} S_{\alpha, \beta}(s) S_{\alpha, \beta}(t) \\
& =g_{\beta+1}(s) J_{t}^{\alpha} S_{\alpha, \beta}(t)-g_{\beta+1}(t) J_{s}^{\alpha} S_{\alpha, \beta}(s)
\end{aligned}
$$

holds for $s, t \geq 0$, where $J_{t}^{\alpha}$ is the Riemann-Liouville fractional integral of order $\alpha$.
The generator $A$ of $S_{\alpha, \beta}(t)$ is defined by

$$
\begin{gathered}
D(A):=\left\{x \in X: \lim _{t \rightarrow 0^{+}} \frac{S_{\alpha, \beta}(t) x-g_{\beta+1}(t) x}{g_{\alpha+\beta+1}(t)} \text { exists }\right\}, \\
A x:=\lim _{t \rightarrow 0^{+}} \frac{S_{\alpha, \beta}(t) x-g_{\beta+1}(t) x}{g_{\alpha+\beta+1}(t)}, \quad x \in D(A) .
\end{gathered}
$$

Note that an $(\alpha, 0)$-ROF is just an $\alpha$-ROF.

In this paper, we firstly show that $(\alpha, \beta)$-ROF satisfies an equality which extends (3) and (7) for $\beta$-integrated semigroup and $\alpha$-ROF, respectively. Then, we consider the inhomogeneous fractional order abstract Cauchy problem

$$
\begin{gathered}
{ }^{c} D_{t}^{\alpha} u(t)=A u(t)+f(t), \quad t \in(0, T), \\
u(0)=x_{0}, \quad u^{\prime}(0)=x_{1},
\end{gathered}
$$

where $1<\alpha<2, T>0, f \in L^{1}((0, T), X)$, and $A$ is assumed to be the generator of an $(\alpha, \beta)$-ROF $S_{\alpha, \beta}(t)$ on $X$. We give the relation between the function $v(t) \stackrel{=}{=} S_{\alpha, \beta}(t) x_{0}+\left(g_{1} *\right.$ $\left.S_{\alpha, \beta}\right)(t) x_{1}+\left(g_{\alpha-1} * S_{\alpha, \beta} * f\right)(t)$ and solution of (10). We also study the problem

$$
\begin{gathered}
{ }^{c} D_{t}^{\alpha} v(t)=A v(t)+g_{\beta+1}(t) x, \quad t>0, \\
v^{(k)}(0)=0, \quad k=0,1, \ldots, N-1,
\end{gathered}
$$

where $\alpha>0, x \in X, N$ is the smallest integer greater than or equal to $\alpha$. We prove that if $A$ generates an exponentially bounded $(\alpha, \beta)$-ROF on $X$ if and only if the problem (11) has a unique exponentially bounded classical solution $v_{x}$ and $A v_{x} \in L_{\text {Loc }}^{1}\left(\mathbb{R}^{+}, X\right)$. If $\alpha \rightarrow 1^{+}, \beta=k \in \mathbb{N}$, our Theorem 13 reduces to Lemma 3.2.10 in [2]. When $\alpha=1, \beta=k$, it is easy to see that our Theorem 15 extends and generalizes Theorem 3.2.13 in [2].

This paper is organized as follows. In Section 2, we provide some preliminaries of the fractional calculus and $(\alpha, \beta)$ ROF. Section 3 is devoted to present an equality characteristic of the $(\alpha, \beta)$-ROF. Finally, as an application of $(\alpha, \beta)$-ROF, we discuss the solutions of fractional abstract Cauchy problem in Section 4.

\section{Preliminary}

Recall that the Riemann-Liouville fractional integral of order $\alpha>0$ of $f$ is defined by

$$
J_{t}^{\alpha} f(t)=\left(g_{\alpha} * f\right)(t)=\int_{0}^{t} g_{\alpha}(t-s) f(s) d s,
$$

and the Caputo fractional derivative of order $\alpha>0$ of $f$ can be written as

$$
{ }^{c} D_{t}^{\alpha} f(t)=\frac{d^{m}}{d t^{m}}\left(g_{m-\alpha} *\left(f(t)-\sum_{k=0}^{m-1} f^{(k)}(0) g_{k+1}(t)\right)\right),
$$

where $m$ is the smallest integer greater than or equal to $\alpha$. For more details in fractional calculus, we refer to $[5,20,21]$. 
The Mittag-Leffler function is defined by

$$
E_{\alpha, \beta}(z)=\sum_{n=0}^{\infty} \frac{z^{n}}{\Gamma(\alpha n+\beta)}, \quad E_{\alpha}(z)=E_{\alpha, 1}(z),
$$

$\operatorname{Re} \alpha>0, \beta, z \in \mathbb{C}$

And if $0<\alpha<2, \beta>0$, then

$$
\begin{aligned}
& E_{\alpha, \beta}(z)=\frac{1}{\alpha} z^{(1-\beta) / \alpha} \exp \left(z^{1 / \alpha}\right)+\varepsilon_{\alpha, \beta}(z), \\
& |\arg z| \leq \frac{1}{2} \alpha \pi, \quad E_{\alpha, \beta}(z)=\varepsilon_{\alpha, \beta}(z), \\
& |\arg (-z)|<\left(1-\frac{1}{2} \alpha\right) \pi,
\end{aligned}
$$

where

$$
\varepsilon_{\alpha, \beta}(z)=-\sum_{n=1}^{N-1} \frac{z^{-n}}{\Gamma(\beta-\alpha n)}+O\left(|z|^{-N}\right) \quad \text { as } z \longrightarrow \infty,
$$

and the $O$-term is uniform in $\arg z$ if $|\arg (-z)| \leq(1-(\alpha / 2)-$ $\epsilon) \pi$.

We now recall some properties of $(\alpha, \beta)$-ROF.

Lemma 2 (see [9, Proposition 3.10]). Let $S_{\alpha, \beta}: \mathbb{R}^{+} \rightarrow B(X)$ be an $(\alpha, \beta)$-ROF generated by $A$. The following assertions hold:

(a) $S_{\alpha, \beta}(t) D(A) \subset D(A)$ and $A S_{\alpha, \beta}(t) x=S_{\alpha, \beta}(t) A x$ for $x \in D(A)$ and $t \geq 0$;

(b) for all $x \in X, J_{t}^{\alpha} S_{\alpha, \beta}(t) x \in D(A)$ and $S_{\alpha, \beta}(t) x=$ $g_{\beta+1}(t) x+A J_{t}^{\alpha} S_{\alpha, \beta}(t) x, t \geq 0$

(c) $x \in D(A)$ and $A x=y$ if and only if $S_{\alpha, \beta}(t) x=$ $g_{\beta+1}(t) x+J_{t}^{\alpha} S_{\alpha, \beta}(t) y, t \geq 0$

(d) $A$ is closed.

Lemma 3 (see [9, Proposition 3.5, Theorem 3.11]). Let $\alpha>0$, $\beta \geq 0$. A generates an $(\alpha, \beta)$-ROF $S_{\alpha, \beta}$ satisfying $\left\|S_{\alpha, \beta}(t)\right\| \leq$ $M e^{\omega t}, t \geq 0$, for some constants $M>0$ and $\omega \geq 0$, if and only if $\left(\omega^{\alpha}, \infty\right) \subset \rho(A)$ and there exists a strongly continuous function $S: \mathbb{R}^{+} \rightarrow B(X)$ such that $\|S(t)\| \leq M e^{\omega t}$ for all $t \geq 0$ and $\int_{0}^{\infty} e^{-\lambda t} S(t) x d t=\lambda^{\alpha-\beta-1} R\left(\lambda^{\alpha}, A\right) x, \lambda>\omega$, for all $x \in X$. Furthermore, $S(t)$ is $S_{\alpha, \beta}(t)$.

Lemma 4 (see [2, Proposition B.6]). Let $U \subset \mathbb{C}$. If function $R: U \rightarrow B(X)$ satisfies $R(\lambda)-R(\mu)=(\mu-\lambda) R(\lambda) R(\mu)$, then there is an operator $A$ on $X$ such that $R(\lambda)=(\lambda I-A)^{-1}$ for all $\lambda \in U$ if and only if $\operatorname{ker} R(\lambda)=\{0\}$.

\section{An Novel Equality Characteristic for $(\alpha, \beta)$-ROF}

The following theorem shows that an $(\alpha, \beta)$-ROF satisfies a functional equation and the treatment bases on the technique of Laplace transform. For convenience, we drop the subscript $\alpha, \beta$ from $\left\{S_{\alpha, \beta}\right\}_{t \geq 0}$ in this theorem.
Theorem 5. Let $\alpha \in \mathbb{R}^{+} \backslash \mathbb{N}_{0}, \beta \in \mathbb{R}^{+}$satisfy $\beta-\alpha>-1$. If $\{S(t)\}_{t \geq 0}$ is an $(\alpha, \beta)-R O F$, then it satisfies the following equality:

$$
\begin{aligned}
\int_{t}^{t+s}(s+t-r)^{\beta-\alpha} S(r) d r-\int_{0}^{s}(s+t-r)^{\beta-\alpha} S(r) d r \\
\quad=\frac{\alpha \Gamma(\beta-\alpha+1)}{\Gamma(1-\alpha)} \int_{0}^{s} \int_{0}^{t} \frac{S\left(r_{1}\right) S\left(r_{2}\right)}{\left(t+s-r_{1}-r_{2}\right)^{1+\alpha}} d r_{1} d r_{2} .
\end{aligned}
$$

Proof. Denote by $L(t, s)$ and $R(t, s)$ the left and right sides of equality (17), respectively, and denote by $f_{a}(t)$ the truncation of $f(t)$ at $a$, that is, $f_{a}(t)=f(t)$ for $0 \leq t \leq a$ and $f_{a}(t)=0$ otherwise.

We will show that the Laplace transform of $L_{a}(t, s)$ and $R_{a}(t, s)$ with respect to $t$ and $s$ is equivalent, and by the uniqueness of Laplace transform, we can get that $L_{a}(t, s)=$ $R_{a}(t, s)$.

Taking Laplace transform of $L_{a}(t, s)$ with respect to $s$ as follows

$$
\begin{aligned}
\widehat{L}_{a}(t, \lambda)= & \int_{0}^{\infty} e^{-\lambda s}\left[\int_{t}^{t+s}(s+t-r)^{\beta-\alpha} S_{a}(r) d r\right. \\
& \left.-\int_{0}^{s}(s+t-r)^{\beta-\alpha} S_{a}(r) d r\right] d s \\
= & \int_{t}^{\infty} S_{a}(r) \int_{r-t}^{\infty} e^{-\lambda s}(s+t-r)^{\beta-\alpha} d s d r \\
& -\int_{0}^{\infty} S_{a}(r) \int_{r}^{\infty} e^{-\lambda s}(s+t-r)^{\beta-\alpha} d s d r \\
= & \int_{t}^{\infty} S_{a}(r) e^{-\lambda(r-t)} \int_{0}^{\infty} e^{-\lambda \tau} \tau^{\beta-\alpha} d \tau d r \\
& -\int_{0}^{\infty} S_{a}(r) e^{-\lambda(r-t)} \int_{t}^{\infty} e^{-\lambda \tau} \tau^{\beta-\alpha} d \tau d r \\
= & \frac{\Gamma(\beta-\alpha+1)}{\lambda^{(\beta-\alpha+1)} \int_{t}^{\infty} S_{a}(r) e^{-\lambda(r-t)} d r} \\
& -e^{\lambda t \widehat{S}_{a}(\lambda) \int_{t}^{\infty} e^{-\lambda \tau} \tau^{\beta-\alpha} d \tau,}
\end{aligned}
$$

then taking Laplace transform with respect to $t$, we have

$$
\begin{aligned}
\widehat{L}_{a}(\mu, \lambda)= & \int_{0}^{\infty} e^{-\mu t}\left[\frac{\Gamma(\beta-\alpha+1)}{\lambda^{(\beta-\alpha+1)}} \int_{t}^{\infty} S_{a}(r) e^{-\lambda(r-t)} d r\right. \\
& \left.-e^{\lambda t} \widehat{S}_{a}(\lambda) \int_{t}^{\infty} e^{-\lambda \tau} \tau^{\beta-\alpha} d \tau\right] d t \\
= & \frac{\Gamma(\beta-\alpha+1)}{\lambda^{(\beta-\alpha+1)}} \int_{0}^{\infty} e^{-\mu t} \int_{t}^{\infty} S_{a}(r) e^{-\lambda(r-t)} d r d t \\
& -\widehat{S}_{a}(\lambda) \int_{0}^{\infty} e^{(\lambda-\mu) t} \int_{t}^{\infty} e^{-\lambda \tau} \tau^{\beta-\alpha} d \tau d t
\end{aligned}
$$




$$
\begin{aligned}
& =\frac{\Gamma(\beta-\alpha+1)}{\lambda^{(\beta-\alpha+1)}} \int_{0}^{\infty} e^{-\lambda r} S_{a}(r) \int_{0}^{r} e^{(\lambda-\mu) t} d t d r \\
& -\int_{0}^{\infty} e^{-\lambda \tau} \tau^{\beta-\alpha} \int_{0}^{\tau} e^{(\lambda-\mu) t} d t d \tau \widehat{S}_{a}(\lambda) \\
& =\frac{\Gamma(\beta-\alpha+1)}{(\lambda-\mu) \lambda^{(\beta-\alpha+1)}} \\
& \times\left(\int_{0}^{\infty} e^{-\mu r} S_{a}(r) d r-\int_{0}^{\infty} e^{-\lambda r} S_{a}(r) d r\right) \\
& -\frac{1}{\lambda-\mu} \\
& \times\left(\int_{0}^{\infty} e^{-\mu \tau} \tau^{\beta-\alpha} d \tau-\int_{0}^{\infty} e^{-\lambda \tau} \tau^{\beta-\alpha} d \tau\right) \widehat{S}_{a}(\lambda) \\
& =\frac{\Gamma(\beta-\alpha+1)}{(\lambda-\mu) \lambda^{(\beta-\alpha+1)}}\left(\widehat{S}_{a}(\mu)-\widehat{S}_{a}(\lambda)\right) \\
& -\frac{1}{\lambda-\mu}\left(\frac{\Gamma(\beta-\alpha+1)}{\mu^{(\beta-\alpha+1)}}-\frac{\Gamma(\beta-\alpha+1)}{\lambda^{(\beta-\alpha+1)}}\right) \widehat{S}_{a}(\lambda) \\
& =\frac{\Gamma(\beta-\alpha+1)}{\lambda-\mu}\left(\lambda^{(\alpha-\beta-1)} \widehat{S}_{a}(\mu)-\mu^{(\alpha-\beta-1)} \widehat{S}_{a}(\lambda)\right) \\
& =\frac{\Gamma(\beta-\alpha+1)}{(\lambda-\mu)(\lambda \mu)^{\beta-\alpha+1}} \\
& \times\left(\mu^{(\beta-\alpha+1)} \widehat{S}_{a}(\mu)-\lambda^{(\beta-\alpha+1)} \widehat{S}_{a}(\lambda)\right) \\
& =\frac{\Gamma(\beta-\alpha+1)}{(\lambda-\mu)(\lambda \mu)^{\beta-\alpha+1}}\left(R\left(\mu^{\alpha}, A\right)-R\left(\lambda^{\alpha}, A\right)\right) \text {, }
\end{aligned}
$$

where the last equality follows from Lemma 3.

On the other hand, observing that

$$
R_{a}(t, s)=\frac{\alpha \Gamma(\beta-\alpha+1)}{\Gamma(1-\alpha)} \int_{0}^{t} \frac{S_{a}(r)}{(t+s-r)^{1+\alpha}} d r * S_{a}(s),
$$

Then taking Laplace transform with respect to $t$ and $s$, respectively, we deduce

$$
\begin{aligned}
\widehat{R}_{a}(t, \lambda)= & \frac{\alpha \Gamma(\beta-\alpha+1)}{\Gamma(1-\alpha)} \\
& \times \int_{0}^{\infty} e^{-\lambda s} \int_{0}^{t} \frac{S_{a}(r)}{(t+s-r)^{1+\alpha}} d r d s \widehat{S}_{a}(\lambda) \\
= & \frac{\alpha \Gamma(\beta-\alpha+1)}{\Gamma(1-\alpha)} \\
& \times \int_{0}^{\infty} e^{-\lambda s}(t+s)^{-\alpha-1} * S_{a}(t) d s \widehat{S}_{a}(\lambda),
\end{aligned}
$$

$$
\begin{aligned}
& \widehat{R}_{a}(\mu, \lambda)=\frac{\alpha \Gamma(\beta-\alpha+1)}{\Gamma(1-\alpha)} \\
& \times \int_{0}^{\infty} e^{-\mu t} \int_{0}^{\infty} e^{-\lambda s}(t+s)^{-\alpha-1} * S_{a}(t) d s d t \widehat{S}_{a}(\lambda) \\
& =\frac{\alpha \Gamma(\beta-\alpha+1)}{\Gamma(1-\alpha)} \\
& \times \int_{0}^{\infty} e^{-\lambda s} \int_{0}^{\infty} e^{-\mu t}(t+s)^{-\alpha-1} * S_{a}(t) d s d t \widehat{S}_{a}(\lambda) \\
& =\frac{\alpha \Gamma(\beta-\alpha+1)}{\Gamma(1-\alpha)} \\
& \times \int_{0}^{\infty} e^{-\lambda s} \int_{0}^{\infty} e^{-\mu t}(t+s)^{-\alpha-1} d t d s \widehat{S}_{a}(\mu) \widehat{S}_{a}(\lambda) \\
& =\frac{\Gamma(\beta-\alpha+1)}{\Gamma(1-\alpha)} \frac{\Gamma(1-\alpha)}{\lambda-\mu}\left(\lambda^{\alpha}-\mu^{\alpha}\right) \widehat{S}_{a}(\mu) \widehat{S}_{a}(\lambda) \\
& =\frac{\Gamma(\beta-\alpha+1)}{\lambda-\mu}\left(\lambda^{\alpha}-\mu^{\alpha}\right) \widehat{S}_{a}(\mu) \widehat{S}_{a}(\lambda) \\
& =\frac{\Gamma(\beta-\alpha+1)}{(\lambda-\mu)}\left(\lambda^{\alpha}-\mu^{\alpha}\right) \\
& \times \frac{1}{(\lambda \mu)^{\beta-\alpha+1}} R\left(\mu^{\alpha}, A\right) R\left(\lambda^{\alpha}, A\right) \\
& =\frac{\Gamma(\beta-\alpha+1)}{(\lambda-\mu)(\lambda \mu)^{\beta-\alpha+1}}\left(R\left(\mu^{\alpha}, A\right)-R\left(\lambda^{\alpha}, A\right)\right) \text {, }
\end{aligned}
$$

where the last equality follows from the resolvent identity. In view of (19), (21), and the uniqueness of Laplace transform, we obtain $L_{a}(t, s)=R_{a}(t, s), t, s \geq 0$. The arbitrariness of $a$ implies $L(t, s)=R(t, s)$ for $t, s \geq 0$.

Remark 6. (a) If $\beta=0$, then $(\alpha, 0)$-ROF $S_{\alpha, 0}(t)$ is an $\alpha$-ROF and the equality (17) degenerates to be equality (7).

(b) If we assume that, for each $x \in X$, the map $t \rightarrow$ $S_{\alpha, \beta}(t) x$ is continuously differentiable on $[0, \infty)$ and the limit of $(\alpha, \beta)$-ROF $S_{\alpha, \beta}(t)$ exists as $\alpha \rightarrow 1^{-}$, then multiplying both sides of (17) with $1-\alpha$ and integrating by parts to the right side of (17) and letting $\alpha \rightarrow 1^{-}$, we can get that (3) is just the limit state of (17).

By Lemma 3, $(\alpha, \beta)$-ROF generated by operator $A$ is exactly operator valued functions whose Laplace transforms are $\lambda^{\alpha-\beta-1} R(\lambda, A)$. In the following theorem, we show that this property corresponds to the functional equation (17) for $S_{\alpha, \beta}(t)$. The proof of this theorem is proved by Ardent [2, proposition 3.2.4] for $\alpha \rightarrow 1^{-}, \beta=k \in \mathbb{N}$. Our proof is different since we could not use the binomial formula as in $[2]$. 
Theorem 7. Let $\widetilde{S}: \mathbb{R}^{+} \rightarrow B(X)$ be a strongly continuous function satisfying $\|\widetilde{S}(t)\| \leq M e^{\omega t}(t \geq 0)$ for some $M, \omega \geq 0$. Let $\alpha \in \mathbb{R}^{+} \backslash \mathbb{N}_{0}, \beta \in \mathbb{R}^{+}$satisfy that $\beta-\alpha>-1$, set

$$
R\left(\lambda^{\alpha}\right):=\lambda^{-\alpha+\beta+1} \int_{0}^{\infty} e^{-\lambda t} \widetilde{S}(t) d t
$$

Then the following assertions are equivalent.

(i) There exists an operator $A$ such that $\left(\omega^{\alpha}, \infty\right) \subset \rho(A)$ and $R\left(\lambda^{\alpha}\right)=\left(\lambda^{\alpha} I-A\right)^{-1}$ for $\lambda>\omega$.

(ii) For $s, t \geq 0$, the equality

$$
\begin{aligned}
\int_{t}^{t+s} & (s+t-r)^{\beta-\alpha} \widetilde{S}(r) d r-\int_{0}^{s}(s+t-r)^{\beta-\alpha} \widetilde{S}(r) d r \\
& =\frac{\alpha \Gamma(\beta-\alpha+1)}{\Gamma(1-\alpha)} \int_{0}^{s} \int_{0}^{t} \frac{\widetilde{S}\left(r_{1}\right) \widetilde{S}\left(r_{2}\right)}{\left(t+s-r_{1}-r_{2}\right)^{1+\alpha}} d r_{1} d r_{2}
\end{aligned}
$$

holds and $\widetilde{S}(t) x=0$ for all $t \geq 0$ implies that $x=0$.

Proof. Assume that (i) holds; then $\left(\omega^{\alpha}, \infty\right) \subset \rho(A),\left(\lambda^{\alpha} I-\right.$ $A)^{-1}=\lambda^{-\alpha+\beta+1} \int_{0}^{\infty} e^{-\lambda t} \widetilde{S}(t) d t$ for $\lambda>\omega$; from Lemma 3 , we know that $\widetilde{S}(t)$ is the $(\alpha, \beta)$-ROF generated by $A$; then Theorem 5 shows that equality (23) holds. It follows from $\left(\omega^{\alpha}, \infty\right) \subset \rho(A)$ and $R\left(\lambda^{\alpha}\right)=\left(\lambda^{\alpha} I-A\right)^{-1}$ for $\lambda>\omega$ that $R\left(\lambda^{\alpha}\right)$ is injective. If $\widetilde{S}(t) x=0$ for all $t \geq 0$, from $R\left(\lambda^{\alpha}\right):=$ $\lambda^{-\alpha+\beta+1} \int_{0}^{\infty} e^{-\lambda t} \widetilde{S}(t) d t$, we have $R\left(\lambda^{\alpha}\right) x=0$; thus $x=0$.

If (ii) is satisfied, similar as the calculations of (19) and (21), we can get that the Laplace transform of the left side and the right side of (17) are

$$
\begin{gathered}
\frac{\Gamma(\beta-\alpha+1)}{(\lambda-\mu)(\lambda \mu)^{\beta-\alpha+1}}\left(R\left(\mu^{\alpha}\right)-R\left(\lambda^{\alpha}\right)\right), \\
\frac{\Gamma(\beta-\alpha+1)}{\lambda-\mu} \frac{\lambda^{\alpha}-\mu^{\alpha}}{(\lambda \mu)^{\beta-\alpha+1}} R\left(\mu^{\alpha}\right) R\left(\lambda^{\alpha}\right),
\end{gathered}
$$

respectively. So,

$$
R\left(\mu^{\alpha}\right)-R\left(\lambda^{\alpha}\right)=\left(\lambda^{\alpha}-\mu^{\alpha}\right) R\left(\mu^{\alpha}\right) R\left(\lambda^{\alpha}\right) .
$$

On the other hand, if $R\left(\lambda^{\alpha}\right) x=0$, by $R\left(\lambda^{\alpha}\right)=$ $\lambda^{-\alpha+\beta+1} \int_{0}^{\infty} e^{-\lambda t} \widetilde{S}(t) d t$ and uniqueness of Laplace transform, we have $\widetilde{S}(t) x=0$ for all $t \geq 0$, then from (ii) we know $x=0$, so, $\operatorname{Ker} R\left(\lambda^{\alpha}\right)=0$, by (25) and Lemma 4, we get the conclusion.

\section{Fractional Abstract Cauchy Problems}

In this section, we study the following inhomogeneous fractional abstract Cauchy problem:

$$
\begin{gathered}
{ }^{c} D_{t}^{\alpha} u(t)=A u(t)+f(t), \quad t \in(0, T), \\
u(0)=x_{0}, \quad u^{\prime}(0)=x_{1},
\end{gathered}
$$

where $1<\alpha<2, T>0, f \in L^{1}((0, T), X), x_{0}, x_{1} \in X, A$ is a linear closed operator.
First, we give the definitions of solutions to (26).

Definition 8. A function $u \in C([0, T) ; X)$ is called a mild solution of (26), if $\left(g_{\alpha} * u\right)(t) \in D(A)$ and $u(t)=x_{0}+t x_{1}+$ $A\left(g_{\alpha} * u\right)(t)+\left(g_{\alpha} * f\right)(t), t \in[0, T)$.

Definition 9. A function $u \in C([0, T) ; X)$ is called a classical solution of (26) if $u$ satisfies the following.
(a) $u \in C([0, T) ; D(A)) \cap C^{1}([0, T) ; X)$.
(b) $g_{2-\alpha} *\left(u-x_{0}-t x_{1}\right) \in C^{2}([0, T) ; X)$.
(c) $u$ satisfies (26).

From the above definitions, it is clear that a classical solution of (26) is a mild solution of it. The following assertion shows that a mild solution of the problem (26) with suitable regularity is also a classical solution.

Theorem 10. Let $u$ be a mild solution of (26) and $f \in$ $C([0, T) ; X)$, if $g_{2-\alpha} *\left(u-x_{0}-t x_{1}\right) \in C^{2}([0, T) ; X)$, and for any $t \in(0, T), g_{\alpha} * u \in L^{1}((0, t), D(A))$; then $u$ is also a classical solution of (26).

Proof. Since $u$ is a mild solution of (26), we have

$$
\begin{gathered}
\left(g_{\alpha} * u\right)(t) \in D(A), \\
u(t)=x_{0}+t x_{1}+A\left(g_{\alpha} * u\right)(t)+\left(g_{\alpha} * f\right)(t), \\
t \in[0, T) .
\end{gathered}
$$

If we denote $w(t):=u(t)-x_{0}-t x_{1}$, then it follows from (27) that

$$
\begin{aligned}
\left(g_{2-\alpha} * w\right)(t) & =g_{2-\alpha} *\left(A\left(g_{\alpha} * u\right)(t)+\left(g_{\alpha} * f\right)(t)\right) \\
& =A\left(g_{2} * u\right)(t)+\left(g_{2} * f\right)(t) .
\end{aligned}
$$

Since $g_{2-\alpha} * w \in C^{2}([0, T) ; X)$, then ${ }^{c} D_{t}^{\alpha} u(t)=$ $\left(d^{2} / d t^{2}\right)\left(g_{2-\alpha} * w\right)(t)$ is well defined, and by (28), we have

$$
\begin{aligned}
{ }^{c} D_{t}^{\alpha} u(t)= & \frac{d^{2}}{d t^{2}}\left(g_{2-\alpha} * w\right)(t) \\
= & \lim _{h \rightarrow 0} \frac{1}{h^{2}}\left[\left(g_{2-\alpha} * w\right)(t)-2\left(g_{2-\alpha} * w\right)(t-h)\right. \\
& \left.\quad+\left(g_{2-\alpha} * w\right)(t-2 h)\right] \\
= & \lim _{h \rightarrow 0} \frac{1}{h^{2}}\left[A\left(g_{2} * u\right)(t)-2 A\left(g_{2} * u\right)(t-h)\right. \\
& \left.+A\left(g_{2} * u\right)(t-2 h)\right] \\
& +\lim _{h \rightarrow 0} \frac{1}{h^{2}}\left[\left(g_{2} * f\right)(t)-2\left(g_{2} * f\right)(t-h)\right. \\
& \left.\quad+\left(g_{2} * f\right)(t-2 h)\right] \\
= & \lim _{h \rightarrow 0} \frac{1}{h^{2}}\left[A\left(g_{2} * u\right)(t)-2 A\left(g_{2} * u\right)(t-h)\right. \\
& \left.+A\left(g_{2} * u\right)(t-2 h)\right]+f(t) .
\end{aligned}
$$


Thus,

$$
\begin{aligned}
\lim _{h \rightarrow 0} \frac{1}{h^{2}}[ & A\left(g_{2} * u\right)(t)-2 A\left(g_{2} * u\right)(t-h) \\
& \left.+A\left(g_{2} * u\right)(t-2 h)\right]={ }^{c} D_{t}^{\alpha} u(t)-f(t) .
\end{aligned}
$$

On the other hand, from the closeness of $A$ and $g_{\alpha} * u \in$ $L^{1}((0, t), D(A))$ for $t \in[0, T)$, by Proposition 1.1.7 in [2], we have

$$
\left(g_{2} * u\right)(t)=\left(g_{2-\alpha} *\left(g_{\alpha} * u\right)\right)(t) \in D(A),
$$

Then from (30) and the closeness of $A$, we obtain

$$
\begin{gathered}
u(t)=\lim _{h \rightarrow 0} \frac{1}{h^{2}}\left[\left(g_{2} * u\right)(t)-2\left(g_{2} * u\right)(t-h)\right. \\
\left.+\left(g_{2} * u\right)(t-2 h)\right] \in D(A), \\
{ }^{c} D_{t}^{\alpha} u(t)=A u(t)+f(t), \quad t \in[0, T) .
\end{gathered}
$$

It is clear that $u(0)=x_{0}, u^{\prime}(0)=x_{1}$. Thus, $u$ is a classical solution of (26).

Lemma 11. Let $1<\alpha<2, f \in L^{1}((0, T), X)$. Suppose $A$ is the generator of an $(\alpha, \beta)$-ROF $S_{\alpha, \beta}(t)$ on $X$ for some $\beta \in \mathbb{R}^{+}$. Then, for everyt $\in[0, T),\left(g_{\alpha-1} * S_{\alpha, \beta} * f\right)(t)$ exists, and $\left(g_{\alpha-1} *\right.$ $\left.S_{\alpha, \beta} * f\right) \in C([0, T), X)$.

Proof. For every $t \in[0, T)$, since $g_{\alpha-1} \in L^{1}\left((0, t), \mathbb{R}^{+}\right), f \in$ $L^{1}((0, t), X)$, we get $g_{\alpha-1} * f \in L^{1}((0, t), X)$, hence, from

$$
\begin{aligned}
\left(g_{\alpha-1} * S_{\alpha, \beta} * f\right)(t) & =\left(S_{\alpha, \beta} * g_{\alpha-1} * f\right)(t) \\
& =\int_{0}^{t} S_{\alpha, \beta}(t-s)\left(g_{\alpha-1} * f\right)(s) d s,
\end{aligned}
$$

we obtain that $\left(g_{\alpha-1} * S_{\alpha, \beta} * f\right)(t)$ exists.

For $h \in \mathbb{R},|h| \ll 1$ and $t+h \in[0, T)$, we have

$$
\begin{aligned}
& \left(g_{\alpha-1} * S_{\alpha, \beta} * f\right)(t+h)-\left(g_{\alpha-1} * S_{\alpha, \beta} * f\right)(t) \\
& =\int_{0}^{t+h} S_{\alpha, \beta}(t+h-s)\left(g_{\alpha-1} * f\right)(s) d s \\
& \quad-\int_{0}^{t} S_{\alpha, \beta}(t-s)\left(g_{\alpha-1} * f\right)(s) d s \\
& =\int_{0}^{t+h}\left(S_{\alpha, \beta}(t+h-s)-S_{\alpha, \beta}(t-s)\right)\left(g_{\alpha-1} * f\right)(s) d s \\
& \quad-\int_{t}^{t+h} S_{\alpha, \beta}(t-s)\left(g_{\alpha-1} * f\right)(s) d s .
\end{aligned}
$$

From the dominated convergence theorem and absolute continuity of integral, we deduce

$$
\lim _{h \rightarrow 0}\left(\left(g_{\alpha-1} * S_{\alpha, \beta} * f\right)(t+h)-\left(g_{\alpha-1} * S_{\alpha, \beta} * f\right)(t)\right)=0 .
$$

So, $\left(g_{\alpha-1} * S_{\alpha, \beta} * f\right) \in C([0, T), X)$.
Let

$$
v(t)=S_{\alpha, \beta}(t) x_{0}+\left(g_{1} * S_{\alpha, \beta}\right)(t) x_{1}+\left(g_{\alpha-1} * S_{\alpha, \beta} * f\right)(t) .
$$

From Lemma 11, we know that $v$ is well defined, and $v \in$ $C([0, T), X)$.

The following theorem is proved by Arendt [2, Lemma 3.2.9] for $\alpha=1, \beta=l \in \mathbb{N}$. Our proof is different because we could not use the formula of integration by parts as [2, Lemma 3.2.9].

Theorem 12. Suppose that $A$ is the generator of an $(\alpha, \beta)-R O F$ $S_{\alpha, \beta}(t)$ on $X$ for some $\beta \in \mathbb{R}^{+}$. Let $v$ be defined by (36). Then one has the following results.

(a) If (26) has a mild solution $u$, then $g_{m-\beta} *(v-$ $\left.\sum_{k=0}^{m-1} v^{(k)}(0) g_{k+1}(t)\right) \in C^{m}([0, T) ; X)$ and $u(t)=$ ${ }^{c} D_{t}^{\beta} v(t)$.

(b) If there is a classical solution $u$ of (26), then $g_{2-\alpha} *$ $\left({ }^{c} D_{t}^{\beta} v(t)-x_{0}-t x_{1}\right) \in C^{2}([0, T) ; X)$ and $u(t)=$ ${ }^{c} D_{t}^{\beta} v(t)$.

Proof. If $u$ is a mild solution of (26), then $\left(g_{\alpha} * u\right)(t) \in D(A)$ and

$u(t)=x_{0}+t x_{1}+A\left(g_{\alpha} * u\right)(t)+\left(g_{\alpha} * f\right)(t), \quad t \in[0, T)$.

Using Lemma 2(b) and the closeness of $A$, we have

$$
\begin{aligned}
\left(g_{\beta+1} * u\right)(t)= & \left(S_{\alpha, \beta}-A\left(g_{\alpha} * S_{\alpha, \beta}\right)\right) * u(t) \\
= & \left(S_{\alpha, \beta} * u\right)(t)-\left(A\left(g_{\alpha} * S_{\alpha, \beta}\right) * u\right)(t) \\
= & \left(S_{\alpha, \beta} * u\right)(t)-S_{\alpha, \beta} * A\left(g_{\alpha} * u\right)(t) \\
= & \left(S_{\alpha, \beta} * u\right)(t) \\
& -S_{\alpha, \beta} *\left(u-x_{0}-t x_{1}-\left(g_{\alpha} * f\right)(t)\right) \\
= & S_{\alpha, \beta} * x_{0}+S_{\alpha, \beta} * t x_{1}+\left(S_{\alpha, \beta} * g_{\alpha} * f\right)(t) ;
\end{aligned}
$$

that is, $\left(g_{\beta+1} * u\right)(t)=\left(1 * S_{\alpha, \beta}\right)(t) x_{0}+\left(g_{2} * S_{\alpha, \beta}\right)(t) x_{1}+\left(g_{\alpha} *\right.$ $S * f)(t)$. So

$$
\begin{aligned}
J_{t}^{\beta} u(t)= & \left(g_{\beta} * u\right)(t)=\frac{d}{d t}\left(g_{\beta+1} * u\right)(t) \\
= & S_{\alpha, \beta}(t) x_{0}+\left(g_{1} * S_{\alpha, \beta}\right)(t) x_{1} \\
& +\left(g_{\alpha-1} * S_{\alpha, \beta} * f\right)(t)=v(t)
\end{aligned}
$$

Thus, it follows from $u \in C([0, T), X)$ that $g_{m-\beta} *(v-$ $\left.\sum_{k=0}^{m-1} v^{(k)}(0) g_{k+1}(t)\right) \in C^{m}([0, T) ; X)$ and $u(t)={ }^{c} D_{t}^{\beta} v(t)$. Hence (a) holds. If $u$ is a classical solution of (26), then $u$ is a mild solution of (26). So, assertion (b) follows immediately from (a). 
Theorem 13. Let $v$ be defined by (36). Assume that $v \in$ $C^{m-1}([0, T) ; X), v^{(k)}(0)=0$ for $k=0,1, \ldots, m-1$, and $g_{m-\beta} * v \in C^{m}([0, T) ; X)$; then ${ }^{c} D_{t}^{\beta} v(t)$ is a mild solution of the problem (26). Moreover, if $g_{2-\alpha} *\left({ }^{c} D_{t}^{\beta} v(t)-x_{0}-\right.$ $\left.t x_{1}\right) \in C^{2}([0, T) ; X)$, and for any $t \in(0, T), g_{\alpha} *{ }^{c} D_{t}^{\beta} v(t) \in$ $L^{1}((0, t), D(A))$, then ${ }^{c} D_{t}^{\beta} v(t)$ is also a classical solution of (26).

Proof. Consider the following steps.

Step 1. We first claim that $J_{t}^{\alpha} v(t) \in D(A)$ and

$$
{ }^{c} D_{t}^{\beta} A J_{t}^{\alpha} v(t)={ }^{c} D_{t}^{\beta} v(t)-x_{0}-t x_{1}-g_{\alpha} * f .
$$

In view of definition of $v(t)$, we have

$$
\begin{aligned}
J_{t}^{\alpha} v(t)= & \left(g_{\alpha} * S_{\alpha, \beta}\right)(t) x_{0}+\left(g_{\alpha} * g_{1} * S_{\alpha, \beta}\right)(t) x_{1} \\
& +\left(g_{\alpha} * g_{\alpha-1} * S_{\alpha, \beta} * f\right)(t) \\
= & \left(g_{\alpha} * S_{\alpha, \beta}\right)(t) x_{0}+\int_{0}^{t}\left(g_{\alpha} * S_{\alpha, \beta}\right)(\tau) x_{1} d \tau \\
& +\int_{0}^{t}\left(g_{\alpha} * S_{\alpha, \beta}\right)(t-\tau)\left(g_{\alpha-1} * f\right)(\tau) d \tau,
\end{aligned}
$$

for $t \in[0, T)$.

From Lemma 2(b), for $0<\tau<t$, we have

$$
\begin{gathered}
\left(g_{\alpha} * S_{\alpha, \beta}\right)(t) x_{0} \in D(A), \quad\left(g_{\alpha} * S_{\alpha, \beta}\right)(\tau) x_{1} \in D(A), \\
\quad\left(g_{\alpha} * S_{\alpha, \beta}\right)(t-\tau)\left(g_{\alpha-1} * f\right)(\tau) \in D(A), \\
A\left(g_{\alpha} * S_{\alpha, \beta}\right)(\tau) x_{1}=S_{\alpha, \beta}(\tau) x_{1}-g_{\beta+1}(\tau) x_{1} \in L^{1}(0, t), \\
A\left(g_{\alpha} * S_{\alpha, \beta}\right)(t-\tau)\left(g_{\alpha-1} * f\right)(\tau) \\
=S_{\alpha, \beta}(t-\tau)\left(g_{\alpha-1} * f\right)(\tau) \\
\quad-g_{\beta+1}(t-\tau)\left(g_{\alpha-1} * f\right)(\tau) \in L^{1}(0, t),
\end{gathered}
$$

combining with the closeness of $A$, one has

$$
\begin{gathered}
\int_{0}^{t}\left(g_{\alpha} * S_{\alpha, \beta}\right)(\tau) x_{1} d \tau \in D(A), \\
\int_{0}^{t}\left(g_{\alpha} * S_{\alpha, \beta}\right)(t-\tau)\left(g_{\alpha-1} * f\right)(\tau) d \tau \in D(A) .
\end{gathered}
$$

Thus $J_{t}^{\alpha} v(t) \in D(A)$, and

$$
\begin{aligned}
A J_{t}^{\alpha} v(t)= & A\left(g_{\alpha} * S_{\alpha, \beta}\right)(t) x_{0}+g_{1} * A\left(g_{\alpha} * S_{\alpha, \beta}\right)(t) x_{1} \\
& +g_{\alpha-1} * A\left(g_{\alpha} * S_{\alpha, \beta} * f\right)(t)
\end{aligned}
$$

$$
\begin{aligned}
= & S_{\alpha, \beta}(t) x_{0}-g_{\beta+1}(t) x_{0}+\left(g_{1} * S_{\alpha, \beta}\right)(t) x_{1} \\
& -\left(g_{1} * g_{\beta+1}\right)(t) x_{1} \\
& +g_{\alpha-1} *\left(S_{\alpha, \beta} * f-g_{\beta+1} * f\right)(t) \\
= & S_{\alpha, \beta}(t) x_{0}+\left(g_{1} * S_{\alpha, \beta}\right)(t) x_{1} \\
& +\left(g_{\alpha-1} * S_{\alpha, \beta} * f\right)(t)-g_{\beta+1}(t) x_{0} \\
& -\left(g_{1} * g_{\beta+1}\right)(t) x_{1}-\left(g_{\alpha+\beta} * f\right)(t) \\
= & v(t)-g_{\beta+1}(t) x_{0}-\left(g_{1} * g_{\beta+1}\right)(t) x_{1} \\
& -\left(g_{\alpha+\beta} * f\right)(t) .
\end{aligned}
$$

So

$$
\begin{aligned}
A J_{t}^{\alpha} v(t)= & v(t)-g_{\beta+1}(t) x_{0}-\left(g_{1} * g_{\beta+1}\right)(t) x_{1} \\
& -\left(g_{\alpha+\beta} * f\right)(t), \\
{ }^{c} D_{t}^{\beta} A J_{t}^{\alpha} v(t)= & { }^{c} D_{t}^{\beta} v(t)-x_{0}-t x_{1}-g_{\alpha} * f .
\end{aligned}
$$

Step 2. We prove ${ }^{c} D_{t}^{\beta} J_{t}^{\alpha} v(t) \in D(A)$ and $A^{c} D_{t}^{\beta} J_{t}^{\alpha} v(t)=$ ${ }^{c} D_{t}^{\beta} A J_{t}^{\alpha} v(t)$.

Since $v \in C^{k}([0, T) ; X), v^{(k)}(0)=0$ for $k=0,1, \ldots, m-1$, we have

$$
\left.\frac{d^{k}}{d t^{k}}\left(g_{\alpha} * v\right)(t)\right|_{t=0}=\left.\left(g_{\alpha} * v^{(k)}\right)(t)\right|_{t=0}=0 .
$$

So

$$
\begin{aligned}
{ }^{c} D_{t}^{\beta} J_{t}^{\alpha} v(t) & =\frac{d^{m}}{d t^{m}}\left(g_{m-\beta} * g_{\alpha} * v\right)(t) \\
& =\lim _{h \rightarrow 0} \frac{1}{h^{m}} \sum_{r=0}^{m} C_{m}^{r}\left(g_{m-\beta} * g_{\alpha} * v\right)(t-r h),
\end{aligned}
$$

where $C_{m}^{r}=(m(m-1) \cdots(m-r+1)) / r$ !. From (45), we know that $A J_{t}^{\alpha} v \in L^{1}((0, t), X)$, and the closeness of $A$ implies that $\left(g_{m-\beta} * g_{\alpha} * v\right)(t) \in D(A)$, and $A\left(g_{m-\beta} * g_{\alpha} * v\right)(t)=g_{m-\beta} *$ $A\left(g_{\alpha} * v\right)(t)$, by Step $1,{ }^{c} D_{t}^{\beta} A J_{t}^{\alpha} v(t)$ exists, then ${ }^{c} D_{t}^{\beta} J_{t}^{\alpha} v(t) \epsilon$ $D(A)$, and

$$
A^{c} D_{t}^{\beta} J_{t}^{\alpha} v(t)={ }^{c} D_{t}^{\beta} A J_{t}^{\alpha} v(t) .
$$

Step 3. We show that $v^{(k)}(0)=0$ for $k=0,1, \ldots, m-1$ implies

$$
{ }^{c} D_{t}^{\beta} J_{t}^{\alpha} v(t)=J_{t}^{\alpha c} D_{t}^{\beta} v(t) .
$$

In fact, if $\alpha \geq \beta$, we have ${ }^{c} D_{t}^{\beta} J_{t}^{\alpha} v(t)=J_{t}^{\alpha-\beta} v(t)$, and

$$
\begin{aligned}
J_{t}^{\alpha c} D_{t}^{\beta} v(t) & =J_{t}^{\alpha-\beta} J_{t}^{\beta c} D_{t}^{\beta} v(t) \\
& =J_{t}^{\alpha-\beta}\left(v(t)-\sum_{k=0}^{m-1} v^{(k)}(0) g_{k+1}(t)\right) .
\end{aligned}
$$


If $\alpha<\beta$, we have ${ }^{c} D_{t}^{\beta} J_{t}^{\alpha} v(t)={ }^{c} D_{t}^{\beta-\alpha} v(t)$, and

$$
\begin{aligned}
J_{t}^{\alpha c} D_{t}^{\beta} v(t) & ={ }^{c} D_{t}^{\beta-\alpha} J_{t}^{\beta c} D_{t}^{\beta} v(t) \\
& ={ }^{c} D_{t}^{\beta-\alpha}\left(v(t)-\sum_{k=0}^{m-1} v^{(k)}(0) g_{k+1}(t)\right) .
\end{aligned}
$$

From the above discussion and $v^{(k)}(0)=0$ for $k=0,1, \ldots$, $m-1$, we conclude that (49) holds.

Finally, in view of (40), (48), and (49), we have

$$
\begin{aligned}
A J_{t}^{\alpha c} D_{t}^{\beta} v(t) & =A^{c} D_{t}^{\beta} J_{t}^{\alpha} v(t)={ }^{c} D_{t}^{\beta} A J_{t}^{\alpha} v(t) \\
& ={ }^{c} D_{t}^{\beta} v(t)-x_{0}-t x_{1}-\left(g_{\alpha} * f\right)(t) .
\end{aligned}
$$

Therefore, ${ }^{c} D_{t}^{\beta} v(t)$ is a mild solution of (26).

Moreover, if $g_{2-\alpha} *\left({ }^{c} D_{t}^{\beta} v(t)-x_{0}-t x_{1}\right) \in C^{2}([0, T) ; X)$, and for any $t \in(0, T), g_{\alpha} *{ }^{c} D_{t}^{\beta} v(t) \in L^{1}((0, t), D(A))$, applying Theorem 10, we have that ${ }^{c} D_{t}^{\beta} v(t)$ is a classical solution of (26).

Remark 14. If $\alpha \rightarrow 1^{+}, \beta=k$, then (26) becomes (4). Theorem 13 degenerated to Lemma 3.2.10 in [2]. Note that the condition $v^{(j)}(0)=0$ for $j=0,1, \ldots, k-1$ is not necessary in Lemma 3.2.10 of [2], since from its proof, it is easy to see that $v(0)=0$ implies that $v^{(j)}(0)=0$ for $j=1, \ldots, k-1$.

Now, we turn our attention to the problem

$$
\begin{aligned}
& { }^{c} D_{t}^{\alpha} v(t)=A v(t)+g_{\beta+1} t(x), \quad t>0, \\
& v^{(k)}(0)=0, \quad k=0,1, \ldots, N-1,
\end{aligned}
$$

where $\alpha>0, x \in X, A$ is a linear closed operator on $X$ and $N$ is the smallest integer greater than or equal to $\alpha$.

Theorem 15. Let $A$ be a closed operator on $X$ and $\beta>0$; the following two assertions are equivalent.

(i) A generates an exponentially bounded $(\alpha, \beta)-R O F S_{\alpha, \beta}$ on $X$.

(ii) For every $x \in X$, there exists a unique classical solution $v_{x}$ of (53) which is exponentially bounded and $A v_{x} \in$ $L_{\text {loc }}^{1}\left(\mathbb{R}^{+} ; X\right)$.

Proof. If $(i)$ is satisfied, for every $x \in X$, define $v_{x}: \mathbb{R}^{+} \rightarrow X$ by $v_{x}(t)=\left(g_{\alpha} * S_{\alpha, \beta}\right)(t) x$, then $v_{x}^{(k)}(0)=0$ for $k=0,1, \ldots, N-$ 1. By Lemma 2(b), we have $v_{x}(t)=\left(g_{\alpha} * S_{\alpha, \beta}\right)(t) x \in D(A)$, and

$$
\begin{aligned}
{ }^{c} D_{t}^{\alpha} v_{x}(t) & =S_{\alpha, \beta}(t) x=A\left(g_{\alpha} * S_{\alpha, \beta}\right)(t) x+g_{\beta+1}(t) x \\
& =A v_{x}(t)+g_{\beta+1}(t) x, \quad t>0 .
\end{aligned}
$$

Thus, $v_{x}$ is a classical solution of (53); it is unique by Theorem 12 . Since $S_{\alpha, \beta}$ is exponentially bounded, we have that $v_{x}$ is exponentially bounded. From

$$
A v_{x}(t)=A\left(g_{\alpha} * S_{\alpha, \beta}\right)(t) x=S_{\alpha, \beta}(t) x-g_{\beta+1}(t) x,
$$

we know that $A v_{x}(t) \in L_{\text {loc }}^{1}\left(\mathbb{R}^{+} ; X\right)$. So (ii) is true.
Assume that (ii) holds. From linearity of (53) and the uniqueness of its solution, we get that $v_{x}$ is linear in $x$. So, for each $t \geq 0$, there exists a linear mapping $V(t): X \rightarrow D(A)$ such that $V(t) x=v_{x}(t)$ for any $x \in X$.

Next, we show that, for each $t \geq 0, V(t) \in B(X, D(A))$.

We consider the mapping $\Phi: X \rightarrow C\left(\mathbb{R}^{+}, D(A)\right)$ by $\Phi(x)=v_{x}(\cdot)=V(\cdot) x$. Then, $\Phi$ is a linear operator defined on $X$. Now we show that $\Phi$ is closed, if $x_{n} \rightarrow x$ in $X$ and $\Phi\left(x_{n}\right) \rightarrow u$ in $C\left(\mathbb{R}^{+}, D(A)\right)$. For $t>0$, by the dominated convergence theorem, we have that $J_{t}^{\alpha} v_{x_{n}}(t)$ converges to $J_{t}^{\alpha} u(t)$, since $v_{x_{n}}(\cdot)=g_{\alpha+\beta+1}(t) x_{n}+J_{t}^{\alpha} A v_{x_{n}}(t)$, from the closeness of $A$, it follows that as $n \rightarrow \infty, u(t)=$ $g_{\alpha+\beta+1}(t) x+J_{t}^{\alpha} A u(t)$, which implies that $u=\Phi(x)$ and $\Phi$ is closed. Therefore, by the closed graph theorem, $\Phi$ is bounded. So, for each $t \geq 0, V(t) \in B(X, D(A))$. Then, the exponentially boundedness of $V(t) x$ and Lemma 3.2.14 in [2], imply that $\|V(t)\| \leq M e^{\omega t}(t \geq 0)$ for some constants $M$, $\omega \geq 0$. So $Q(\lambda) x=\lambda^{\beta+1} \int_{0}^{\infty} e^{-\lambda t} V(t) x d t$ is well defined for $\lambda>\omega,\left(\omega^{\alpha}, \infty\right) \subset \rho(A)$.

Since $A V(t) x \in L^{1}\left(\mathbb{R}^{+}, X\right)$, then the Laplace transform of $A V(t) x$ is well defined, and from the closeness of $A$, for $\lambda>\omega$, we have

$$
\begin{aligned}
\left(\lambda^{\alpha}-A\right) Q(\lambda) x= & \lambda^{\alpha+\beta+1} \int_{0}^{\infty} e^{-\lambda t} V(t) x d t-\lambda^{\alpha+\beta+1} \\
& \times \int_{0}^{\infty} e^{-\lambda t} A V(t) x d t \\
= & \lambda^{\alpha+\beta+1} \int_{0}^{\infty} e^{-\lambda t} V(t) x d t-\lambda^{\beta+1} \\
& \times \int_{0}^{\infty} e^{-\lambda t^{c}} D_{t}^{\alpha} V(t) x d t+\lambda^{\beta+1} \\
& \times \int_{0}^{\infty} e^{-\lambda t} g_{\beta+1}(t) x d t \\
= & \lambda^{\alpha+\beta+1} \widehat{V}(\lambda) x-\lambda^{\beta+1} \lambda^{\alpha} \widehat{V}(\lambda) x \\
& +\lambda^{\beta+1} \lambda^{-(\beta+1)} x=x .
\end{aligned}
$$

Now, we show that $\left(\lambda^{\alpha}-A\right)$ is injective for $\lambda>\omega$. Assume that $\left(\lambda^{\alpha}-A\right) x=0$ for some $x \in D(A)$ and $\lambda>\omega$. Then, by the method of Laplace transform, we have that the solution of (53) is $t^{\alpha+\beta} E_{\alpha, \alpha+\beta+1}\left(\lambda^{\alpha} t^{\alpha}\right)$. Since $\left\|v_{x}(t)\right\| \leq M e^{\omega t}$, for all $t \geq 0$, combine with (15), it follows that $x=0$. Hence $\left(\lambda^{\alpha}-A\right)^{-1}=$ $Q(\lambda)$ for $\lambda>\omega$ and $V(t)$ is an $(\alpha, \alpha+\beta)$-ROF. Let

$$
S_{\alpha, \beta}(t) x:={ }^{c} D_{t}^{\alpha} V(t) x=A V(t) x+g_{\beta+1}(t) x ;
$$

then $S_{\alpha, \beta}(t) x$ exists and $V(t) x=J_{t}^{\alpha} S_{\alpha, \beta}(t) x$ for all $t \geq 0$ and all $x \in X$. So

$$
S_{\alpha, \beta}(t) x=A J_{t}^{\alpha} S_{\alpha, \beta}(t) x+g_{\beta+1}(t) x,
$$

and taking the Laplace transform, we have

$$
\widehat{S}_{\alpha, \beta}(\lambda) x=A \lambda^{-\alpha} \widehat{S}_{\alpha, \beta}(\lambda) x+\lambda^{-\beta-1} x, \quad \lambda>\omega ;
$$


that is,

$$
\widehat{S}_{\alpha, \beta}(\lambda) x=\lambda^{\alpha-\beta-1}\left(\lambda^{\alpha}-A\right)^{-1} x, \quad \lambda>\omega .
$$

From Lemma 3, we know that $S_{\alpha, \beta}$ is the $(\alpha, \beta)$-ROF generated by $A$.

Remark 16. Theorem 15 extends and generalizes Theorem 3.2.13 in [2]. In fact, when $\alpha=1$ and $\beta=k$, (53) becomes (5), $S_{\alpha, \beta}(t)$ is a $k$-times integrated semigroup. For problem (5), the condition $A v_{x} \in L_{\text {loc }}^{1}\left(\mathbb{R}^{+} ; X\right)$ in (ii) is not necessary. Since from the proof of Theorem 3.2.13 in [2], it is easy to see that the assumption that exponentially boundedness of the unique classical solution to the problem (5) imply that $A v_{x} \in L_{\text {loc }}^{1}\left(\mathbb{R}^{+} ; X\right)$.

\section{Acknowledgments}

This work is supported by the Program for New Century Excellent Talents in University (NECT-12-0246) and FRFCU (lzujbky-2013-k02).

\section{References}

[1] M. Mijatović, S. Pilipović, and F. Vajzović, " $\alpha$-times integrated semigroups $\left(\alpha \in \mathbb{R}^{+}\right)$," Journal of Mathematical Analysis and Applications, vol. 210, no. 2, pp. 790-803, 1997.

[2] W. Arendt, C. J. K. Batty, M. Hieber, and F. Neubrander, VectorValued Laplace Transforms and Cauchy Problems, vol. 96 of Monographs in Mathematics, Birkhäuser, Basel, Switzerland, 2001.

[3] T. E. Duncan, Y. Hu, and B. Pasik-Duncan, "Stochastic calculus for fractional Brownian motion. I. Theory," SIAM Journal on Control and Optimization, vol. 38, no. 2, pp. 582-612, 2000.

[4] R. Hilfer, Applications of Fractional Calculus in Physics, World Scientific, Singapore, 2000.

[5] A. A. Kilbas, H. M. Srivastava, and J. J. Trujillo, Theory and Applications of Fractional Differential Equations, vol. 204 of North-Holland Mathematics Studies, Elsevier Science, Amsterdam, The Netherlands, 2006.

[6] V. E. Tarasov, Fractional Dynamics: Applications of Fractional Calculus to Dynamics of Particles, Fields and Media, Springer, New York, USA, 2010.

[7] J. Wang and Y. Zhou, "A class of fractional evolution equations and optimal controls," Nonlinear Analysis: Real World Applications, vol. 12, no. 1, pp. 262-272, 2011.

[8] J. Wang, Y. Zhou, and W. Wei, "Fractional sewage treatment models with impulses at variable times," Applicable Analysis, vol. 92, pp. 1959-1979, 2013.

[9] C. Chen and M. Li, "On fractional resolvent operator functions," Semigroup Forum, vol. 80, no. 1, pp. 121-142, 2010.

[10] M. Kostić, “ $(a, k)$-regularized $C$-resolvent families: regularity and local properties," Abstract and Applied Analysis, vol. 2009, Article ID 858242, 27 pages, 2009.

[11] M. Li, C. Chen, and F.-B. Li, "On fractional powers of generators of fractional resolvent families," Journal of Functional Analysis, vol. 259, no. 10, pp. 2702-2726, 2010.

[12] L. Kexue and P. Jigen, "Fractional abstract Cauchy problems," Integral Equations and Operator Theory, vol. 70, no. 3, pp. 333361, 2011.
[13] K. Li, J. Peng, and J. Jia, "Cauchy problems for fractional differential equations with Riemann-Liouville fractional derivatives," Journal of Functional Analysis, vol. 263, no. 2, pp. 476-510, 2012.

[14] C. Lizama, "An operator theoretical approach to a class of fractional order differential equations," Applied Mathematics Letters, vol. 24, no. 2, pp. 184-190, 2011.

[15] C. Lizama, "Regularized solutions for abstract Volterra equations," Journal of Mathematical Analysis and Applications, vol. 243, no. 2, pp. 278-292, 2000.

[16] G. da Prato and M. Iannelli, "Linear integro-differential equations in Banach spaces," Rendiconti del Seminario Matematico dell'Università di Padova, vol. 62, pp. 207-219, 1980.

[17] E. Bajlekova, Fractional evolution equations in Banach spaces [Ph.D. thesis], Eindhoven University of Technology, 2001.

[18] J. Peng and K. Li, "A novel characteristic of solution operator for the fractional abstract Cauchy problem," Journal of Mathematical Analysis and Applications, vol. 385, no. 2, pp. 786-796, 2012.

[19] J. Prüss, Evolutionary Integral Equations and Applications, Birkhäuser, Basel, Switzerland, 1993.

[20] K. S. Miller and B. Ross, An Introduction to the Fractional Calculus and Fractional Differential Equations, John Wiley \& Sons, New York, NY, USA, 1993.

[21] I. Podlubny, Fractional Differential Equations, vol. 198 of Mathematics in Science and Engineering, Academic Press, San Diego, Calif, USA, 1999. 


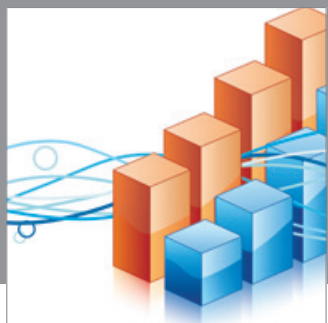

Advances in

Operations Research

mansans

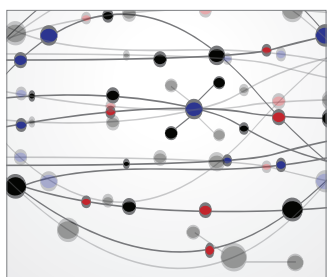

The Scientific World Journal
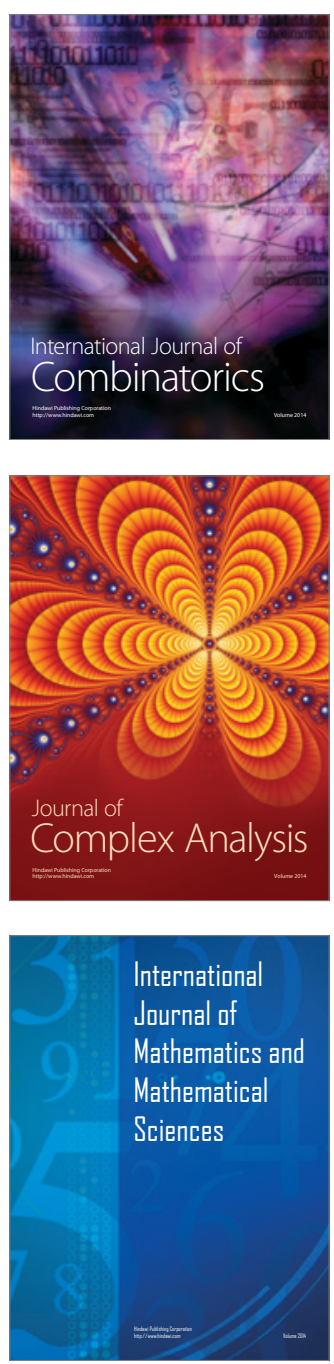
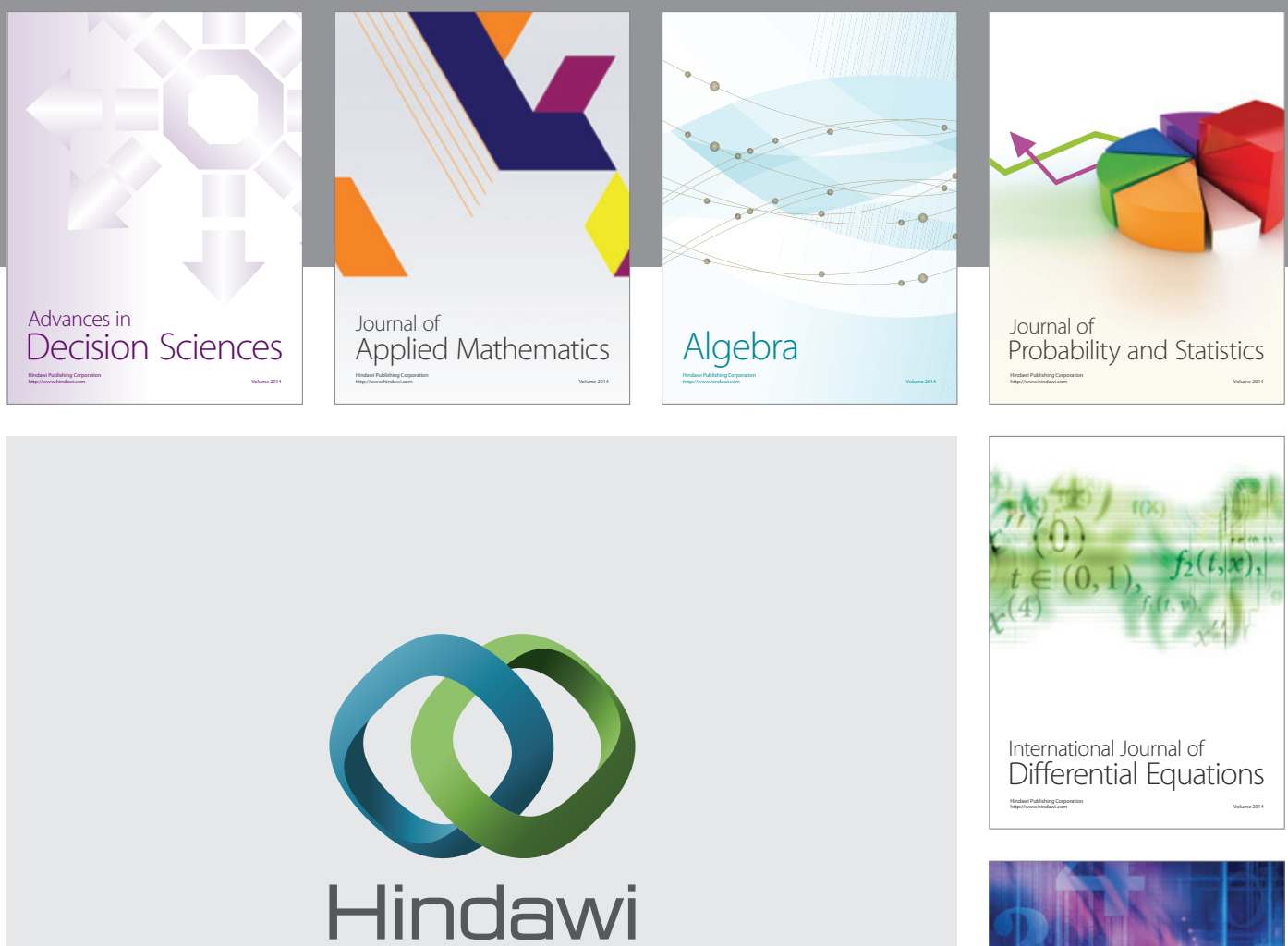

Submit your manuscripts at http://www.hindawi.com
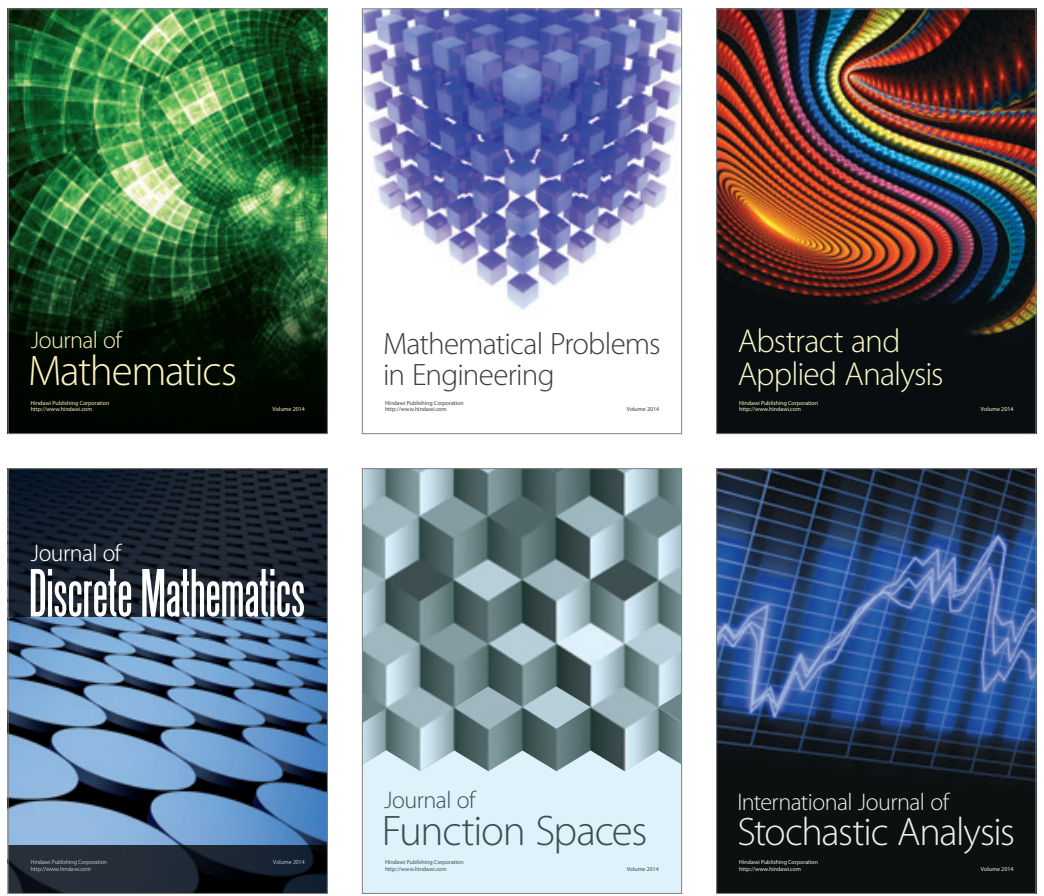

Journal of

Function Spaces

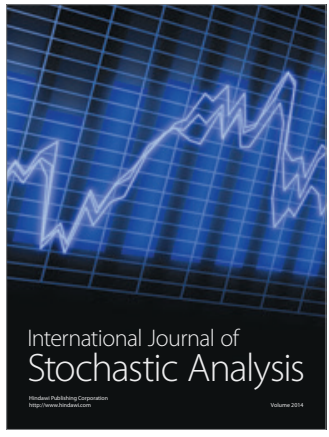

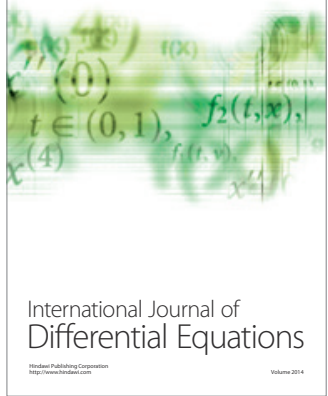
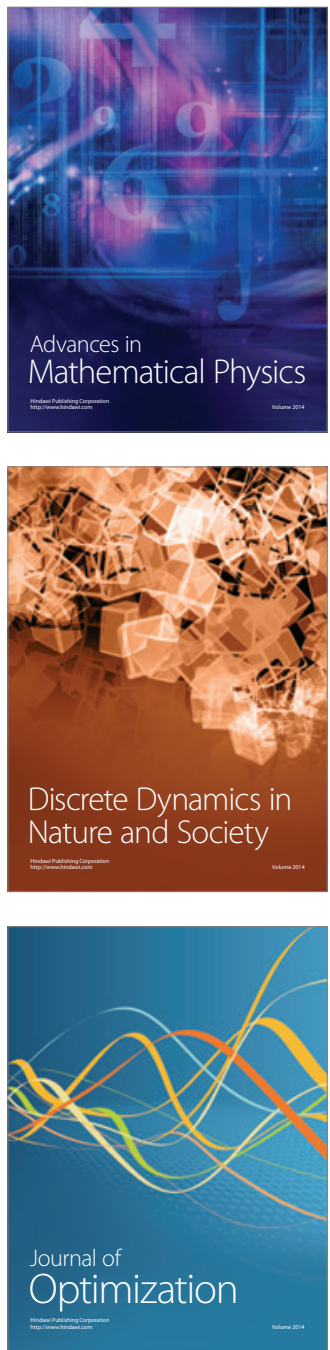